\title{
From Translation to Navigation of Different Discourses: A Model of Search Term Selection during the Pre-Online Stage of the Search Process
}

\author{
Mirja livonen \\ Department of Information Studies and Sociology, University of Oulu, P.O. Box 111, FIN-90571 Oulu, Finland. \\ E-mail: mirja.iivonen@oulu.fi \\ Diane H. Sonnenwald* \\ School of Information and Library Science, CB \#3360, University of North Carolina at Chapel Hill, Chapel Hill, \\ NC 27599-3360. E-mail: dhs@ils.unc.edu
}

\begin{abstract}
We propose a model of the search term selection process based on our empirical study of professional searchers during the pre-online stage of the search process. The model characterizes the selection of search terms as the navigation of different discourses. Discourse refers to the ways of talking and thinking about a certain topic; there often exists multiple, diverse discourses on the same topic. When selecting search terms, searchers appear to navigate a variety of discourses, i.e., they view the topic of a client's search request from the perspective of multiple discourse communities, and evaluate and synthesize differences and similarities among those discourses when selecting search terms. Six discourses emerged as sources of search terms in our study. These discourses are controlled vocabularies, documents and the domain, the practice of indexing, clients' search requests, databases, and the searchers' own search experience. Data further suggest that searchers navigate these discourses dynamically and have preferences for certain discourses. Conceptualizing the selection of search terms as a meeting place of different discourses provides new insights into the complex nature of the search term selection process. It emphasizes the multiplicity and complexity of sources of search terms, the dynamic nature of the search term selection process, and the complex analysis and synthesis of differences and similarities among sources of search terms. It suggests that searchers may need to understand fundamental aspects of multiple discourses in order to select search terms.
\end{abstract}

* To whom all correspondence should be addressed.

Received November 12, 1996; revised February 7, 1997; accepted March 14, 1997

(C) 1998 John Wiley \& Sons, Inc.

\section{Introduction}

The selection of search terms is one of the key processes in information retrieval. However, it is not well understood and remains a key issue and problem (Fidel, 1991a). Traditionally, the selection of search terms has been conceptualized and described as a translation process. In the translation model, a search request, provided by a client, is "translated" into search terms. The search terms, ideally, represent search concepts or search topics that can be input to an information retrieval (IR) system to identify documents, i.e., information-bearing items such as books, articles, video, audiotapes, etc., relevant to the client's information need (see e.g., International Organization for Standardization, 1985; Lancaster, 1972).

The translation process is, in practice, often operationalized as the replacement of one word with another, i.e., a client's word is replaced one-for-one with a search term. However, a client's information need or topic may be discussed in information sources, and represented in IR systems, with a variety of words or phrases. The translation model does not encourage searchers-either professional searchers or end-users - to generate multiple search terms, or consider that a topic may be discussed and represented multiple ways in information sources and IR systems. Considering the variety of ways a topic may be discussed and represented is becoming increasingly important for several reasons. Information in many fields (or domains) is expanding rapidly; multi- and cross-disciplinary information needs are increasing in number and importance as multi-disciplinary work and project teams increase; and information searching environments includ- 
ing the Internet are becoming increasingly diffuse and complex. As Hjerppe (1996) points out, the Internet is very dynamic and contains many diverse documents that are very difficult to control. Searchers may need to consistently generate new search terms that reflect the changing nature of information available on the Internet.

In this article, we propose a model of the search term selection process based on our empirical study of professional searchers during the pre-online stage of the search process. Although the study concentrates on the pre-online stage of the search process, search term selection is a critical issue during the entire search process. Our model characterizes the selection of search terms not only as a translation process, but also as a navigation among different discourses. That is, the selection of search terms may be viewed as a process where searchers step into various discourses and encounter, or discover, different ways of talking about the same topic. Search terms may be viewed as a meeting place, or crossroads, of various discourses, i.e., those places where concepts intersect in some way.

Discourse refers to the ways of talking and thinking about a certain topic within a community (Abercrombie, Hill, \& Turner, 1994). Discourses are not unique or stable. There may exist different discourses of the same topic concurrently. Further, the discourses of a topic may change over time. Discourses also have social functions; they have been produced in social practice and also influence social practice. For example, the information retrieval research community has its own discourse that includes concepts and terms, such as users, relevance feedback, vector space models, probabilistic models, cosines, data fusion, data visualization, etc. These concepts and terms are used when discussing and thinking about information retrieval in the information science research community. The professional library community has a somewhat different discourse and uses concepts and terms, such as clients, subject headings and checkings, to discuss information retrieval. Of course, there is overlap among concepts and terms between the IR research and professional library discourses. Nonetheless, there are also differences between them. The differences and similarities will persist as the two communities practice and develop their discourses in separate and overlapping social forums.

The results of our research suggest that when searchers select search terms to describe a certain search topic, they may step through various discourses in which this topic may be discussed and conceptualized differently. Searchers in our study navigated through six different discourses: The discourse of controlled vocabularies, the discourse of documents and the domain, the discourse of indexing practice, the discourse of clients' search requests, the discourse of databases, and the searcher's own previous search experiences. The searchers selected and navigated these discourses dynamically. Some discourses were preferred over others. In practice, not every dis- course may be navigated for every search request. Depending on the client's need, a given discourse may not be required, a searcher may not have the knowledge or skills required to traverse a discourse, and/or time and resources may not permit such thoroughness. These results suggest that searchers may need to understand fundamental aspects of multiple discourses in order to select search terms, particularly in the pre-online stage of search term selection. This has implications for professional searchers and educators, IR system designers and, ultimately, clients or end-users of IR systems.

\subsection{Related Studies}

Although few studies investigate human actors' decisions in the selection of search terms, several recent studies indicate that the selection of search terms is more than a translation process between the client's search request and IR system-specific search terms. These studies also indicate that professional searchers select search terms from a variety of sources and may use "rules" to help generate alternative search terms.

In their study of four professional searchers, each conducting 10 searches in an academic environment, Saracevic, Mokros, Su, and Spink (1991; see also Spink \& Saracevic, 1997) analyzed clients' written search requests, videotapes, and transcripts of the interaction between each client and the professional searcher, search logs that captured human-computer interaction sequences between each professional searcher and the IR system, and clients' relevance judgments of retrieved items. They found that $38 \%$ of all search terms used came from the written search requests; an additional $23 \%$ were suggested by clients during the on-line search process with a professional searcher. Thirty-nine percent of all search terms were derived from sources other than the client. That is, $19 \%$ were derived from a thesaurus; $11 \%$ from term relevance feedback (when relevant items were retrieved, their examination suggested new terms); and the professional searcher suggested $9 \%$ of all search terms. Spink and Saracevic (1997) found that search terms which were based on clients' written search requests or suggested by clients during the on-line search process were most effective in retrieving relevant documents (as determined by the clients).

Shute and Smith (1993) investigated search term selection by a professional searcher. They found that the searcher used knowledge about the domain to refine users' information needs and generate search terms. They suggest an expert system should also use knowledge about the domain to help users formulate search terms.

Fidel (1986, 1991a, 1991b, 1991c) illustrated that searchers consider various alternatives for search terms on the basis of their previous experience and their present work environment. Based on observation and analysis of verbal and search protocols from 47 professional search- 
ers, Fidel proposed that the process of selecting search terms could be formalized in "rules" or decision trees. She proposed a series of rules to help determine when descriptors and/or free-text words should be used as search terms. She also found that the searchers who worked in different types of search environments (practical, theoretical, and general) appeared to use different subsets of these rules.

Bates (1990) considered various ways of dividing the labor of the information search process between person and system. She pointed out that in a search process there are different moves, tactics, stratagems, and strategies. A move is an identifiable thought or action that is a part of information searching. A tactic is one or more moves made to further the search process. A stratagem consists of multiple tactics and/or moves. A strategy is a plan, which contains moves, tactics, and/or stratagems, for an entire information search. Some of the tactics (super, sub, relate, rearrange, contrary, respell, and respace) mentioned by Bates are related to the selection of search from various sources. Later Bates, Wilde, and Siegfried (1993) found clear differences in search terms selected by scholars with different backgrounds. They noticed that humanities scholars tended to use geographical, chronological, and individual names while physical scientists used mainly subject and common names.

Iivonen (1994, 1995) investigated the differences in the selection of search terms among 32 professional searchers. She found that intersearcher inconsistency, i.e., differences in search terms selected for the same query statement, was partly caused by the fact that searchers considered different sources for search terms.

These studies suggest that the selection of search terms can be a complex process; searchers may consider different sources of search terms, and individual searchers consider these sources differently.

\subsection{Research Objectives}

Therefore, our research objective is to explain more fully the search term selection process, and individual differences in this process. Through the development of a model of the search term selection process, we aim to provide insights into guidelines for searching practice and education, as well as for the design of IR systems. In this article, we begin by proposing a model of the pre-online stage of the search term selection process.

\section{Research Approach: An Empirical Study}

To develop a model, we studied how 32 searchers with different backgrounds individually conceptualized the search term selection process during the pre-online search term selection process. In our study, each searcher was presented with 12 queries that were based on actual search requests received in various libraries in Finland. Each searcher individually formulated a query statement for a commercial IR system for each search request, and afterwards was asked to explain how they generated each query statement.

\subsection{Study Participants}

The study participants included 24 professional searchers from three types of search environments and eight information studies students at a Finnish university. The professional searchers, who worked for different organizations in Finland, had at least 2 years of current professional experience in information retrieval. In their organizations, the professional searchers would normally receive client requests similar to the search requests used in the study, i.e., each searcher worked in an organization where they performed searches in the field of social science. The professional searchers included: (a) nine searchers who worked in special service-oriented search environments including special libraries and two university libraries offering services to specific user groups in social science subject areas; (b) seven searchers who worked in academically-oriented search environments including university libraries and an information bureau offering services to academics throughout Finland; and (c) eight searchers who worked in public search environments, e.g., large public libraries.

The study participants who worked in special serviceoriented search environments had used external databases for 3 years, on average. They typically used only a few external information services, and they typically conducted approximately 40 subject searches per month for clients.

Participants who worked in academically-oriented search environments had used external databases for 14 years on average. In addition, they used several external information services including international services. The number of subject searches performed for clients in a month was approximately 77 , but varied considerably from one organization to another, ranging from 40 to 220 .

Participants from public search environments had used external databases for approximately 7 years. In particular, they used those databases available in Finnish IR systems. The number of subject searches they typically performed in a month was 55 , on average.

The study also included eight undergraduate students in information studies. All the students had completed 10 study weeks (approximately equivalent to 10 credits in U.S. schools ) in information storage and retrieval. In their courses, they had received both theoretical foundations and practical training in database structures and searching.

\subsection{Search Requests}

Each study participant analyzed 12 search requests and formulated query statements for them. These search re- 
quests are listed in Appendix A. All were based on real questions received at various libraries, but not at those libraries where our study participants worked. The search requests were selected from requests on current topics frequently discussed in the mass media. Each search request came from the social sciences, primarily political science. The searchers received the search requests in writing to guarantee that all searchers received identical requests. The searchers were told that in all cases the client was a student whose intention was to write a graduate-level seminar paper.

\subsection{Search Term Selection}

Study participants formulated query statements for the Finnish database KOTI, which is part of the Finnish IR system KDOK/MINTTU. The KOTI database contained 183,760 references at the time of the study. With this system it is possible to use both index terms (descriptors) from Yleinen suomalainen asiasanasto (The General Finnish Thesaurus) and free-text terms as search terms. The only way to combine search terms was to use Boolean operators (due to the constraints of the KDOK/MINTTU system). After generating the query statements, each searcher was asked to explain their query statements, including their selection of search term concepts and search terms. Searchers were encouraged to speak freely and explain their query statements in their own way. For example, the searchers were not asked if they selected search terms from a variety of sources; this result emerged during data analysis. All explanations provided by the study participants were recorded on audiotape and transcribed.

\subsection{Data Analysis}

The searchers' explanations were analyzed using both open coding and axial coding (Berg, 1989). During open coding, the data, i.e., transcripts of the explanations provided by the study participants, were read thoroughly and carefully, and the basic concepts and categories were identified. For example, a basic concept that emerged from the data focused on differences in sources of search terms. Sources of search terms that were mentioned by the searchers were then recognized as categories, or coding frames. These categories are illustrated in Appendix B. The categories were further used in the following stage of axial coding. During axial coding, the data were reread and organized according to the categories. It was then possible to count how many searchers generated search terms from various discourses, and analyze differences and similarities among the different searchers.

\subsection{Study Limitations}

This study focused on the pre-online search phase of search term selection and did not investigate the online stage per se or user-intermediary interaction. We chose this approach for several reasons. We propose, as do other researchers, that IR systems should support actors', or users', cognitive processes. That is, ideally, systems should reflect and support actors' cognitive models. One way to discover these models is to study how actors conceptualize the search term process in general, before they face specific human-computer interaction issues. ${ }^{1}$ Furthermore, searching is increasingly done by individual users, for example, via the World Wide Web or networked IR systems that deliver online searching capabilities to people's desktops. Thus, we chose to first focus on the selection of search terms by individuals, before they interact with an IR system, to increase our understanding of the cognitive processes they bring to bear on the problem. Future research includes studying search term selection during the online process and the influence of user-intermediary interaction on search term selection.

In this study, we also did not collect data on search term effectiveness. Clients were not asked to provide relevance judgments on documents because our data was collected during the pre-online stage of the search process. We focused on the selection of search terms as a cognitive process in order to develop a more comprehensive model of this process; further research is required to determine how aspects of the model contribute to search effectiveness.

\section{Sources of Search Terms: Results from the Empirical Study}

The results of the data analysis indicate that when searchers select search terms, they appear to do more than translate the client's search request into the words of a controlled vocabulary. From the searchers' own explanations, it was possible to see that they navigated, i.e., reflected on ways of thinking and talking about a topic in, a variety of discourses. The six discourses that emerged are: Controlled vocabularies, documents and the domain, indexing practice, client's search requests, databases, and their previous search experience. As illustrated in Figure $1,93.8 \%$ (30 out of 32 ) searchers discussed controlled vocabulary to explain their selection of search terms; $71.9 \%$ (23 out of 32) searchers discussed documents to explain their selection; $68.8 \%$ (22 out of 32 ) discussed the practice of indexing; $43.8 \%$ (14 out of 32 ) discussed clients' words; and $31.3 \%$ (10 out of 32 ) discussed the database and their search experience to explain their selection of search terms.

\subsection{Controlled Vocabulary}

A controlled vocabulary is a fixed list of terms used in an IR system to describe documents. It usually includes

\footnotetext{
${ }^{1}$ Of course, in practice, actors' cognitive models are influenced by IR systems, and our resulting model also includes actors' perceptions of IR systems.
} 

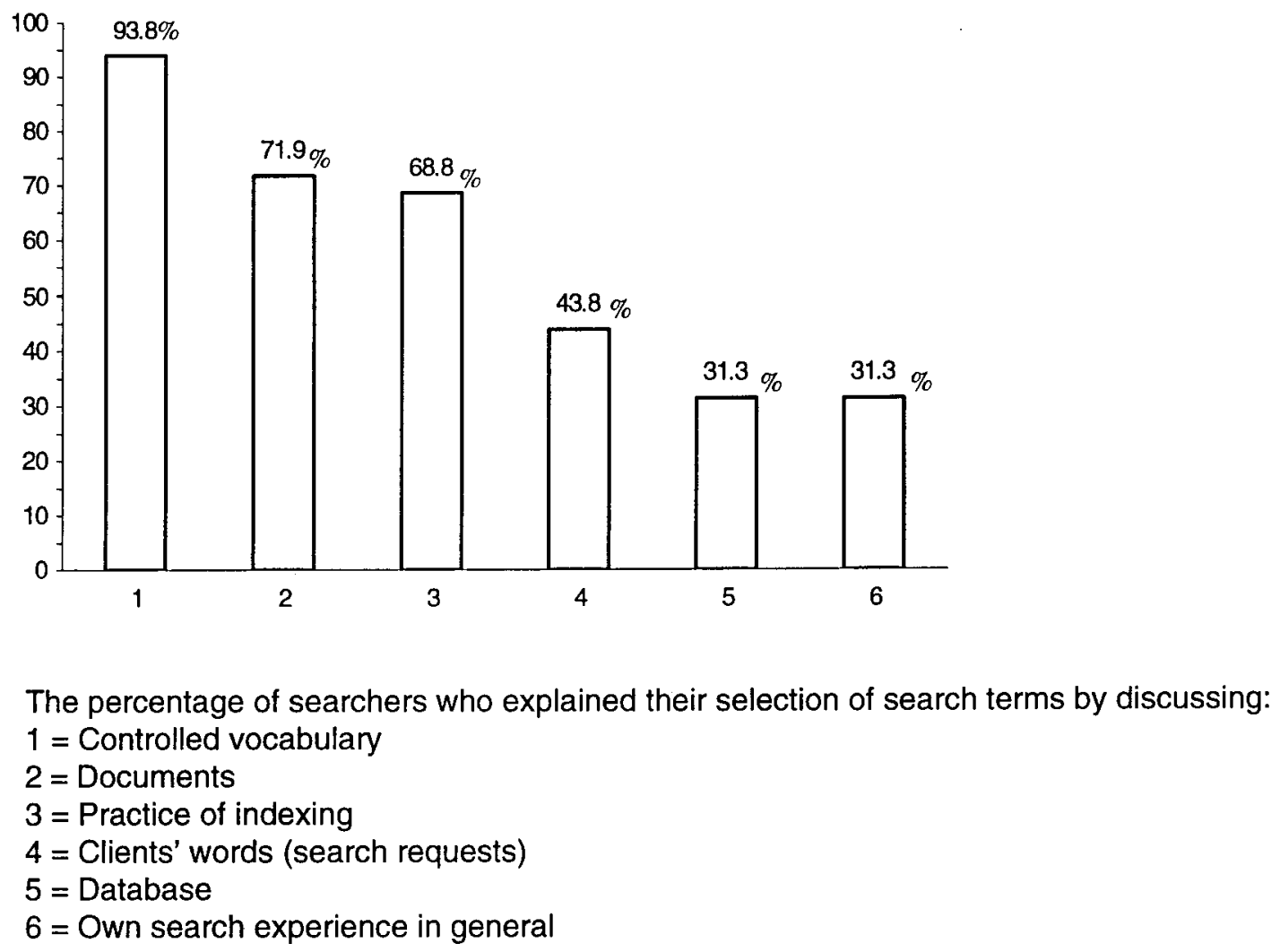

FIG. 1. Discourses navigated when selecting search terms.

the relationships between terms and sometimes the rules for the use of the vocabulary, e.g., how terms should be combined to express compound concepts such as "Finnish political system.' 'Further, the vocabulary may provide rules about how to decompose words not found in the vocabulary, e.g., proper names, into vocabulary terms. The controlled vocabulary is a discourse; it offers a frame through which the world can be interpreted and described. It also restricts alternative ways of talking about topics very clearly, and often very strongly, because terms and their relationships have been standardized. A controlled vocabulary is built through social practice; many professionals review and contribute to it.

In our study, the searchers' knowledge of controlled vocabulary ${ }^{2}$ played a very important role for the searchers as proposed by the translation model. Almost every searcher (30 searchers out of 32 or $93.8 \%)^{3}$ referred to

\footnotetext{
${ }^{2}$ To enhance the readability of this text, sources of search terms are often referred to only by their descriptors. That is, the "searchers' knowledge of controlled vocabulary" is simply referred to as "controlled vocabulary" and "the searchers' knowledge of documents and the domain" is simply referred to as "documents and the domain," etc.

${ }^{3}$ Because we did not conduct a think-aloud study but relied on the memory of study participants, an aggregate unit of analysis, i.e., searchers, was used. We cannot ascertain how accurately searchers recalled their thought processes when selecting terms for each and every search request. However, we can determine general trends.
}

it when they justified their selection of search terms (see Fig. 1). For example, one searcher reported that she liked to use a controlled vocabulary; for one search request she found a specific but unusual search term, "North Pole," in the vocabulary. (Only three searchers out of 32 used this search term.) She described her selection of this unusual search terms as follows:

(Search request: The naval strategies of the U.S. and the Soviet Union with respect to military security of Northern Seas.)

I found from the vocabulary this kind of smart descriptor, "North Pole." Using only "'northern"' would have been too general. (ASE-1) ${ }^{4}$

Another searcher, who selected many various search terms to describe the search concept "social problems," found them from the controlled vocabulary. She justified her selection the following way:

(Search request: Social problems, alienation from society,

\footnotetext{
${ }^{4}$ We use the code ASE for searchers who worked in academicallyoriented search environments, SSE for searchers who worked in special service-oriented search environments, PSE searchers who worked in public search environments, and STE for students. The numbers distinguish various searchers in the same search environment type.
} 
and structural change in society, and their influence on the control people have over their everyday lives in an affluent society.)

This was an extremely broad subject with so many possibilities. So, I found one search term, "social problems", from the vocabulary. Yes, there are others that are narrower terms for social problems; social problems is just a broader term. I was sure that alone it is not enough. So I picked up several narrower terms from the vocabulary although I left some out. I selected from the vocabulary "alienation, isolation, unemployment, homelessness, alcohol problems, drug problems, poverty.", (SSE-1)

These examples illustrate that the controlled vocabulary is, in a very concrete way, present in the selection of search terms. As a discourse, the controlled vocabulary offers ways to talk about a certain topic and how to navigate within topics. At the same time, however, it restricts other alternatives very clearly and often very strongly. In the controlled vocabulary, there always exists the function of standardization; both the preferred terms and their relationships have been standardized. By showing the relationships between terms, the controlled vocabulary can also define concepts for searchers (as illustrated in the previous example). It does not matter if the concept definition is correct or wrong, the concept is defined in any case. For example, it is possible that concepts and relationships between concepts are defined differently among controlled vocabularies. However, the definitions and relationships are given, i.e., fixed, in each vocabulary and cannot be changed; searchers must use them "as is."

\subsection{Documents and the Domain}

Documents are not only information-bearing items, but can be viewed as artifacts of a discourse the author wishes to conduct across time and/or distance. A document's title is a brief description of that discourse. Documents' titles and abstracts (when available) are from a domain discourse. They represent how a certain topic is discussed in text within a community of authors and publishers. There can be many ways of discussing the same topic. However, an author's title and abstract usually reflects only one particular way. Documents and their titles were also an important source for search terms in our study. Twenty-three searchers $(71.9 \%)$ referred to documents and their titles in their justification of search terms. To select search terms, they thought about the ways that authors of documents wrote and thought about the topics mentioned in the search requests. For example, for a search request where social problems and people's everyday lives in society were discussed, one searcher selected search terms "welfare', (in Finnish, "hyvinvointi"') and "'welfare state", (in Finnish, "hyvinvointivaltio", ). These search terms were not mentioned in the search request. However, the searcher justified her selection as follows:
(Search request: Social problems, alienation from society, and structural change in society, and their influence on the control people have over their everyday lives in an affluent society)

I first thought that it [the term, welfare] may occur in a title or in a subtitle of a book [for] this kind of topic. . . . This [search request] is just about the context [of the welfare state]. It is about the welfare state. If I could find an author or a book from that area, I would offer it to the client. (SSE-2)

Because documents' full text, i.e., the documents' own words, and information about their domains are, in many cases, not included in bibliographic databases, the searchers reported trying to imagine which kind of titles they could find in a certain domain. As one searcher explained:

(Search request: Islam in international politics) I imagined titles such as "The Position of Islam in International Politics," "The Role of Muslims." . . . Now I do not remember others, but I had several titles in mind. (SSE-3)

In a certain way, it is a challenge for searchers to explore those other words with which the topic of a search request may be discussed in documents. It is a real task to find an appropriate context within the literature in a domain for a search request.

\subsection{Indexing Practice}

Indexing practice can be described as those work routines, procedures, rules, and restrictions followed in the indexing process including indexing policy and specific decisions made by indexers. This practice of indexing shapes and is shaped by discourse created through formal and informal discussions among professionals at professional conferences and seminars, and in articles and books, etc. Studies of indexing practice (e.g., Iivonen, 1990; Leonard, 1977; Markey, 1984) have shown that professional indexers working in different organizations may use the same controlled vocabulary differently. Thus, we propose that a controlled vocabulary is one discourse, and the practice of indexing, i.e., how a controlled vocabulary is used, is another. Although the practice of indexing may vary between organizations, each practice reflects a particular way of thinking about topics, relationships among topics, and relationships among documents and topics. The discourse of indexing practice favors specific ways of talking about documents and topics, while not accepting other ways.

In our study, the searchers were heavily involved in the discourse of indexing practice. Many searchers (22 out of 32 , or $68.8 \%$ ) mentioned assumptions related to the practice of indexing when they justified the selection of search terms. They thought how indexers would have used index terms in describing certain topics. One 
searcher considered the possible hierarchical levels of terms selected by indexers:

(Search request: Women as candidates, votes received by women candidates and women's voting behavior in Finland in parliamentary elections in the 1980s)

I also took this term, "elections," just to be sure. We may assume that a book is about women in elections in general, e.g., in presidential elections, in local elections, and in parliamentary elections. . . Maybe it is not indexed by the term "parliamentary elections". . . (SSE-4)

The practice of indexing produces ways of talking about a certain topic, and these ways may differ from the ways produced by the authors of the documents, or even the controlled vocabulary. All alternatives offered in the controlled vocabulary are not used in practice. It is understandable that the searchers take the practice of indexing into account when they select search terms.

\subsection{Clients' Search Request}

A search request is a description of the client's information need. The description is generally created by a client and presented to the searcher to initiate the search process. The search request may be expressed either in a written or spoken form. As shown in other studies (e.g., Saracevic et al., 1991) searchers use the search request to locate information that, ideally, satisfies the client's information need. Spink and Saracevic (1997) identified terms from the client's search request as the most effective in retrieving relevant documents.

When clients describe a search request in their own words, they bring their own discourse, or way of communicating about a topic, to the search process. The clients' words are not necessarily very familiar to professional searchers. Searchers may, however, trust the clients' ways of talking about the topic and consider the clients' words as search terms. When clients name a certain concept with a certain word (or group of words) they potentially communicate more than the topic of a search request. It may also inform the searcher about the domain and tradition from which the client comes. This is because topics and concepts are often discussed with different words within different traditions. For example, the concept "'user-system interaction" found in the information systems community is also referred to as "human-computer interaction" in the human factors and psychology communities, as "man-machine interaction"' in the engineering community, and as "user interfaces" in the computer science community. Similarly the same word may represent different concepts in different communities. For example, the word "operation" represents different concepts in medicine ( surgery), military (e.g., Operation De- sert Storm), mathematics (multiplication is an operation), and manufacturing (a work process).

In our data, slightly under half of the searchers (14 out of 32 , or $43.8 \%$ ) indicated that they selected certain words for search terms because they were mentioned in a search request. The words of search requests were not always considered good ones by the study participants, but they were, nonetheless, used by the study participants. As one searcher explained:

(Search request: Islam in international politics)

I would say that these words are not good ones, not at all. But I used this "international politics"' in any case because it was in a search request. (STE-1)

Another searcher described her confidence in the client's words as follows:

(Search request: The influence that alternative activities and environmental movements have on social decisionmaking and the use of power, and on solving global environmental problems )

This was difficult. I selected "environmental movements" and "alternative activities." I would not have noticed that "alternative activities.". . . I think that if the search request could have been formulated in another way, I would not have noticed it. . . . I would have used "environmental movements", and "nature conservation movements.” (PSE-1)

As these examples illustrate, it is not always easy for searchers to accept a client's discourse. A client's discourse may introduce a new way of talking about a topic and, hence, new search terms to be considered.

\subsection{Database}

Databases are collections of data stored in formats which allow the data to be retrieved and manipulated. It is important to note that various databases have different contents and indexing structures. For example, many databases in commercial IR systems contain information judged by the providers to pertain to certain subject categories or to be relevant to their market niche. Furthermore, some bibliographic databases include document titles, author, language, year of publication, publisher, and keywords/descriptors; others also include text abstracts. The decisions regarding the content and structure of databases are usually made by systems analysts and marketing executives or managers. Thus, the database represents perspectives on a topic, including what should be included in the topic area, what types of information are important within a topic area and how users should access this information. In this sense, the database can be viewed as constructing a discourse, a way of interacting and perceiving the aboutness of documents. Therefore, a database may 
influence the selection of search terms because the database represents a discourse.

The data from our study illustrated that the database influences and helps frame the selection of search terms. However, somewhat surprisingly to us, it was not cited as a strong influence. Only 10 searchers $(31.3 \%)$ referred to the database when they justified their selection of search terms. They considered whether a certain search term could be, or could not be, useful in the database, or if they would need many or only a few search terms in searching the database. One searcher described this situation as follows:

(Search request: The naval strategies of the U.S. and the Soviet Union with respect to the military security of the Northern seas )

This is also a topic from which there does not exist so much; at least in the KOTI-database, there does not exist so many documents. Therefore, I tried to find many alternatives for search terms. (ASE-2)

\subsection{Previous Search Experience within Discourses}

For some people, expertise in searching (or any other skill) may be built through practical experience. The practical search experience of professional searchers is shaped by access to, and selection and frequency of use of, different databases and different IR systems. It is also shaped by their exposure to controlled vocabularies, documents, indexing practice, clients' search requests, and discussions among searchers. It is obvious that this experience may form a way of thinking and talking about the selection of search terms. It may define a set of rules that describe ways of constructing search terms (see, e.g., Fidel 1991a, 1991b, 1991c). These rules provide some methods of search term construction and eliminate others. Searchers' previous experience may be conceptualized as a discourse that they may navigate when selecting search terms.

Surprisingly, only 10 searchers $(31.3 \%)$ in our study referred to their own previous search experience when they justified the selection of search terms, although 24 subjects out of 32 had a minimum of 2 years professional search experience. One searcher described the effect of her own search experience as follows:

(Search request: Social problems, alienation from society, and structural change in society, and their influence on the control people have over their everyday lives in an affluent society.)

I once searched about alienation and noticed that it is impossible to find anything about it. Somehow it was the only exact social-psychological term in the request and I knew already beforehand that it does not work. This gave me a bit of a headache. (PSE-2)
One might expect that searchers would also mention formal knowledge about the search process when describing why they selected certain search terms, in addition to mentioning their experience. However, this was not the case. In addition, the low percentage of searchers explicitly mentioning or reflecting on their previous search experience within discourses was surprising. There are several possible explanations for this. One is methodological. It has been noticed that it is difficult for professionals to describe aloud the effect of their own experience on their performance (Schön, 1983). Perhaps searchers in this study implicitly made assumptions based on their previous experience and just did not make the connection between experience and decision making explicit. ${ }^{5}$ Alternatively, we must consider that the dynamic nature of professional searching, including the diversity of search requests, may provide minimal opportunity for searchers to generalize across searches. Or, perhaps we do not teach or sufficiently emphasize this skill in our courses on professional searching.

Previous search experience produces ways of thinking about topics, and these ways may differ from the way the clients think about topics. Further research is required to determine if formal knowledge about the search process also provides a discourse to explore when selecting search terms.

\subsection{Selection of Search Terms in the Different Types of Search Environments}

Differences in the influence of different discourses in the selection of search terms emerged between searchers working in the different types of search environments (see Table 1). Although the number of participants from each environment is too small to determine if the differences between search environments are statistically significant, several trends appear to emerge from the results.

Only the controlled vocabulary was frequently referred to as a source of search terms in each type of search environment. This illustrates the strong position of the discourse of controlled vocabularies for professional searchers. The searchers working in academically-oriented and special service-oriented search environments took into account documents and their titles more often than the students or the searchers working in public search environments. The collections of documents in academically-oriented and special service-oriented search environments are more limited than in public search environments where all possible subject areas are included. It may therefore be easier for the searchers in these environments to think about the discourse of documents. Possibly

\footnotetext{
${ }^{5}$ This may indicate that previous search experience should not be considered a separate source of search terms. However, we leave it as a separate category until this issue has been resolved.
} 
TABLE 1. Discourses navigated by searchers working in different types of search environments.*

Type of search environments

\begin{tabular}{|c|c|c|c|c|c|c|c|c|c|c|}
\hline \multirow[b]{2}{*}{ Discourse } & \multicolumn{2}{|c|}{$\begin{array}{l}\text { Special service- } \\
\text { oriented search } \\
\text { environment }\end{array}$} & \multicolumn{2}{|c|}{$\begin{array}{l}\text { Academically- } \\
\text { oriented search } \\
\text { environment }\end{array}$} & \multicolumn{2}{|c|}{$\begin{array}{l}\text { Public search } \\
\text { environment }\end{array}$} & \multicolumn{2}{|c|}{$\begin{array}{c}\text { Student } \\
\text { environment }\end{array}$} & \multicolumn{2}{|c|}{$\begin{array}{c}\text { All search } \\
\text { environments }\end{array}$} \\
\hline & $(n=9)$ & $(\%)$ & $(n=7)$ & $(\%)$ & $(n=8)$ & $(\%)$ & $(n=8)$ & $(\%)$ & $(n=32)$ & $(\%)$ \\
\hline Controlled vocabulary & 9 & 100.0 & 6 & 85.7 & 7 & 87.5 & 8 & 100.0 & 30 & 93.8 \\
\hline Documents & 8 & 88.9 & 6 & 85.7 & 5 & 62.5 & 4 & 50.0 & 23 & 71.9 \\
\hline Practice of indexing & 7 & 77.8 & 6 & 85.7 & 5 & 62.5 & 4 & 50.0 & 22 & 68.8 \\
\hline Clients' words & 2 & 22.2 & 5 & 71.4 & 4 & 50.0 & 3 & 37.5 & 14 & 43.8 \\
\hline Database & 1 & 11.1 & 3 & 42.9 & 5 & 62.5 & 1 & 12.5 & 10 & 31.1 \\
\hline Own search experience & 2 & 22.2 & 4 & 57.1 & 4 & 50.0 & 0 & 0 & 10 & 31.1 \\
\hline
\end{tabular}

$* n=$ number of searchers who referred to this discourse.

the restricted collections help searchers to understand and get a specific picture of the literature of the subject area of the search requests. The discourse of clients was referred to mostly by the searchers working in academically-oriented search environments. The clients in this type of search environment were experts in their own domain. It is therefore easy to understand why searchers would respect their clients' discourse and take it into account when selecting search terms.

The professional searchers referred more often to the practice of indexing and to their own search experience than the students. The students did not yet have work experience-either in the area of information storage or information retrieval - and so they could not use it in the selection of search terms. The searchers' own work experience affects their decisions, and when they lack this experience in a discourse, they cannot navigate it.

On the basis of the searchers' justifications of search terms, we find that the searchers know about discourses which contain different terms and use terms differently. Their abilities to take these sources into account appear to vary. Further research is necessary to determine if the differences noted here will be statistically significant for larger, diverse populations.

\section{Discussion}

\subsection{A Meeting Place of Different Discourses}

We propose that the selection of search terms can be conceptualized as a meeting place of different discourses (see Fig. 2). In our study, searchers appeared to navigate several different discourses during the pre-online stage of the search process. It seems plausible that searchers will continue to navigate these discourses, and maybe others, during the online stage of the search process. It appears obvious that a topic of a search request is discussed differently in controlled vocabulary than in other discourses, e.g., documents. A controlled vocabulary usually rules out alternative ways of talking about the topic. It also appears obvious that the practice of indexing may have certain rules and ways of describing topics which clearly differ from clients' discourse. Many times it is even difficult for clients to understand in which way, and why the topic is described just this way in indexing.

When constructing queries, professional searchers appear to do more that just translate, replacing words in a search request one-for-one with words from another discourse. Searchers appear to be more like explorers in different discourses; they know that the same topic, or concept, may be conceptualized and talked about differently in various discourses. They appear to think about candidates for search terms from the different perspectives of discourses, and evaluate and synthesize these perspectives. For example, one searcher described her evaluation and synthesis of search terms as follows:

(Search request: National groups and ethnic conflicts in the Third World and their influence on the activities of international organizations.)

Then [I thought about] the "Third World." Another name is "developing countries." The [controlled] vocabulary advises to use the term "developing countries" instead of the "Third World.', But I am not sure if the indexer would have wanted to use the descriptor "developing countries" when the book is about the Third World. So I selected, as a first alternative, the free-text terms "third" and "world" with truncations; and as a second alternative, "developing countries,', both as a descriptor and a free-text term. . . . Then I selected this " national groups" as a descriptor. I thought they are groups in any case. In a free-text, they may be anything - it is difficult to guess - so we can be satisfied with the descriptors. The text is indexed in any case. There are the descriptors "'national groups,", "ethnic minorities," "'minorities," and "population groups". (ASE-3)

Another searcher described her navigation between different discourses as follows: 


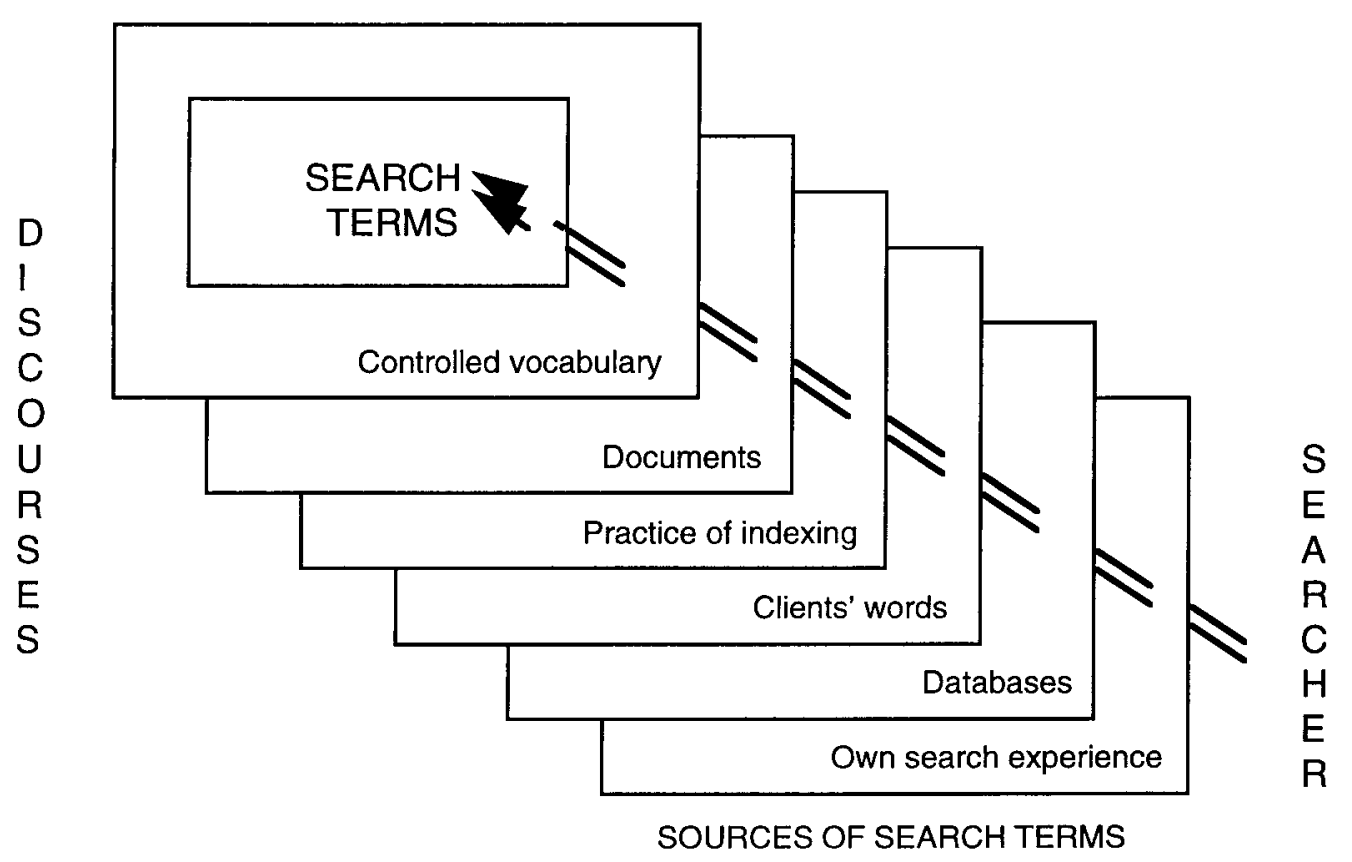

FIG. 2. The selection of search terms as a meeting place of different discourses.

(Search request: The naval strategies of the U.S. and the Soviet Union with respect to military security of the Northern seas.)

For describing this military part of the search request, I selected the descriptors "naval forces," "naval defense," " "naval war," and "naval strategies.'. . . I do not know if they are good ones, but I thought that "naval strategies"' is not enough. Therefore, I also added "naval forces," "naval defense," and "naval war." I do not know if this is the point of view of the request. . . . I thought about the topic and that it may be written with these other words as well. And I do not know how it is indexed. . . . I suppose that the client knows about the topic-it is his idea-and that this word "strategies" may have been used [in documents.] (STE-2)

According to Bloom's (1956) taxonomy of cognitive skills, evaluation and synthesis are the most difficult skills to master. The evaluation and synthesis of perspectives among multiple discourses when selecting and combining search terms may be no different. The search term selection process, like many geographic explorations, may take surprising twists and turns, and not be a linear or sequential process.

\subsection{The Process of Traversing Discourses}

Traversing multiple discourses during the search term selection process does not have to be a linear or sequential process. We propose that searchers may traverse discourses dynamically, moving between discourses oppor- tunistically ( see Fig. 3), based on individual preferences and availability of resources within each discourse. As illustrated in the first quote in the previous section, one searcher began by considering controlled vocabulary and the practice of indexing. Next, the searcher considered documents, and concluded by considering the controlled vocabulary again. As illustrated in the second quote in the previous section, another searcher considered controlled vocabulary, documents, and clients' words and documents, in that order. These examples illustrate that not all searchers will traverse all discourses. It is also possible that a searcher will take into account only one discourse. For example, a novice searcher may not have sufficient knowledge about a database to be able to consider how the database could influence the selection of search terms. In addition, some searchers may traverse discourses not identified in our study.

The dynamic traversal of discourses is consistent with findings by Spink and Saracevic (1997) where no pattern among sources of search terms was found. It is also consistent with decision-making models in other domains. For example Sonnenwald (1993), Sonnenwald and Pejtersen (1994) and Rasmussen, Pejtersen, and Goodstein (1994) observed small groups in naturalistic settings making design decisions. They found that during the decision-making process, the group would dynamically shift among discourses related to design. No patterns in these shifts were discernible.

Further investigation, including think-aloud experiments, may more fully describe sequences of events during the search term selection process during the pre-online 


\section{Discourses}

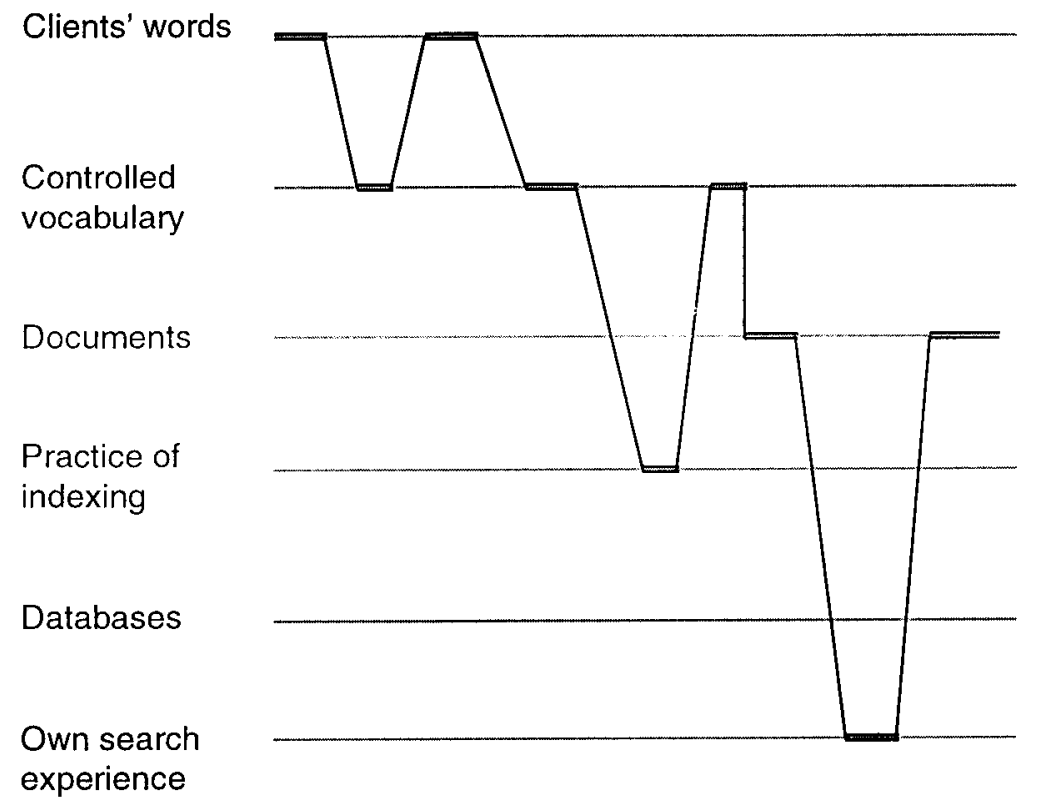

FIG. 3. Searchers appear to traverse discourses dynamically during the search term process.

and online stages. As Simon (1981) pointed out, the path traversed by an ant across a sandy beach appears random when viewed from above (a bird's-eye view). When viewed from the perspective of the ant, where the peaks and valleys of the sandy surface are visible, the ant's path looks quite rational and efficient.

\subsection{Preferences of Discourses}

Although this is a preliminary study, the data suggest that there are several different discourses that are considered as sources for search terms during the pre-online search phase. Some discourses did not appear to be perceived as important as others, or equally considered by all searchers ( see Table 1).

Controlled vocabularies appear to have a strong position in the selection of search terms in general; 93.8\% of the searchers mentioned controlled vocabularies when explaining their selection of search terms. This was followed by documents $(71.9 \%)$, indexing practice $(68.8 \%)$, clients' search requests $(43.8 \%)$, databases $(31.1 \%)$, and previous search experience $(31.1 \%)$. The popularity of a discourse may indicate its authority, accessibility, perceived utility, and familiarity as perceived by professional searchers. For example, controlled vocabularies are taught extensively in library and information study programs, and tools such as controlled vocabularies are usually readily available to searchers.
Documents and domain knowledge often are assigned a high level of status in our profession and culture. Documents, in particular titles and abstracts, are read frequently by searchers in the course of their work. This may help searchers become knowledgeable about the discourse of documents in a domain and the domain itself, because behind documents, there are always domains; documents are part of a domain. Previous work or school experience and/or interaction with domain experts may further increase their knowledge about a domain.

Indexing practice is also a component of a searcher's professional education. However, indexing practice is complex, and its results may be unpredictable. Guides to indexing practice may not be as readily accessible to searchers as controlled vocabularies and documents. Therefore, indexing practice does not appear to have the same preference as controlled vocabularies, as it was mentioned less frequently as a source of search terms than controlled vocabulary and documents.

The next discourse mentioned most frequently as a source of search terms was the discourse of the client as represented by clients' search requests. It was somewhat surprising that the clients' discourse was ranked fourth. This may be explained by the variety and number of clients' discourses. It may be difficult for searchers to synthesize this variety as compared to a controlled vocabulary or document set or indexing practice, which have positions of higher authority in the library and informa- 
tion-service culture. Another explanation for searchers not mentioning clients' words is that the fact that clients were not present when the searchers selected the search terms. In other studies, when clients' words were used more frequently (e.g., Spink \& Saracevic, 1997), clients were present. It is obvious that the presence of a client during the search process would help a professional searcher explore the client's discourse and select search terms from the client's discourse, and this will impact the outcomes of the search process. A client's willingness to participate in the search process and give relevance feedback judgments provide additional ways to navigate, or explore, a discourse. For example, instead of making assumptions about the meaning of a client's words, the searcher can ask the client clarifying questions and explore the client's discourse in ways not possible without the client being there.

Databases appear to be conceptually farther distant from the searcher's professional world than the other discourses discussed thus far. Traditionally, databases have been designed and developed by computer scientists, system analysts, and/or marketing executives. As a result, the discourse of databases may have little in common with the discourse of professional searchers. This is not unique to libraries, information services, and searching, of course; a technology-user gap has been observed in many fields.

The source mentioned least by searchers was previous search experience. We do not wish to suggest that searchers are not reflective practitioners. As discussed earlier, this infrequency may be due to limitations of the research method or a difficulty in generalizing across diverse search experiences.

The preferences for discourses that emerged in this study may then, in part, be explained by social aspects of the discourses. For example, that status ascribed to various discourses by our profession or society, in general, and which can be affected by the presence (or lack thereof) of individuals, may play a role in the navigation of discourses.

A limitation of our study is that we did not explore the effectiveness of search terms. Further research is needed to determine the relationship between search term effectiveness and the navigation of discourses. That is, how does the searcher's preference of, or ability to navigate, discourses influence search results as perceived by clients. Spink and Saracevic (1997) have begun by identifying the effectiveness of search term sources used by four professional searchers. Other recent research, including Su (1991, 1996), Barry (1994), and Schamber and Bateman (1996) has begun to identify a variety of criteria that clients appear to use when evaluating search results. Building on these results, we can begin to compare differences between the navigation of a variety of discourses and search effectiveness, as measured by criteria important to clients. Interestingly, the question of whether differences in the way searchers navigate various discourses effects search term effectiveness, as measured by criteria important to clients, remains open.

\subsection{Implications}

A model of the search term selection process that extends the traditional translation model is particularly relevant today because the complexity of the professional searcher's task is increasing. Documents in any given field (or domain) are now expanding so rapidly that no one individual can keep up with the literature in a field. In addition, clients' needs are increasingly varied and dynamic as individuals and corporations attempt to succeed in the global "Information Age." Databases and search tools are increasing in number and variety. In the Internet environment, the changes are even larger and occur more frequently than in the traditional online search environments. Furthermore, controlled vocabularies are continually updated with word changes, and our practice of indexing is changing as new research on indexing emerges. In an era of corporate downsizing and budget cutbacks, professional searchers may need to change positions and, consequently, change specialties more frequently than before. In sum, professional searchers are faced today with the difficult task of managing an expanding base of information, diverse search requests, and an increasing variety of search tools.

The model presented in this article proposes that the pre-online stage of the search term selection process can be viewed as the navigation of multiple discourses, including controlled vocabulary, documents and the domain, the practice of indexing, clients' words, databases, and searchers' previous experience. This suggests that searchers may need to understand fundamental aspects of multiple discourses in order to select search terms. Consider, for example, the discourse of databases. Table 2 illustrates several aspects of the discourse of databases that may be important to understand when selecting search terms and concepts.

Perhaps professional searchers should, ideally, know what a database is and its goals, constraints, principles of interaction, and hidden assumptions with respect to information. For example, when searchers understand the principles of standard query language (SQL) and first order predicate logic (FOL), they can create complex search statements in most (if not all) commercial databases today. These databases translate all queries into SQL or FOL statements. Of course, SQL and FOL may be difficult to learn, and some commercial databases have interfaces whose purpose is to shield the user from the complexities of SQL and FOL. However, the fact remains that the basis from which the databases operate, or generate search queries, is SQL and FOL. When searchers understand this, they may be able to generate search terms and concepts for any number of databases, and can do so 
TABLE 2. Proposed aspects of the discourse of databases important in the selection of search terms.

\begin{tabular}{|c|c|}
\hline Aspect & Examples from the discourse of databases \\
\hline Membership/definition & $\begin{array}{l}\text { A set of related files, records, data fields, and characters, e.g., OCLC, } \\
\text { INSPEC, KOTI, etc. }\end{array}$ \\
\hline Goals & $\begin{array}{l}\text { To "match" a character string with previously-stored character strings; a } \\
\text { match is done via algorithms that compare characters, sets of characters, } \\
\text { and/or attributes of characters }\end{array}$ \\
\hline Constraints & Only a part of all information possible is available in any particular database \\
\hline Principles of interaction & $\begin{array}{l}\text { For query formulation-standard query language (SQL) and first order } \\
\text { predicate logic (FOL); For human-computer interaction-graphical user } \\
\text { interface (GUI) guidelines and standards }\end{array}$ \\
\hline $\begin{array}{l}\text { Hidden assumptions with } \\
\text { respect to information }\end{array}$ & Information is structured data (Borland, 1987) \\
\hline
\end{tabular}

perhaps better than any database interface, with its very limited (or no) knowledge of the client's needs and documents, ever could. In the future, databases may be based on vector space or probabilistic retrieval models and will, of course, not translate user queries into SQL statements. Perhaps understanding these algorithms and internal representations would also help professional searchers navigate databases.

This approach may be repeated for each discourse that searchers navigate when selecting search terms. For example, understanding clients' goals with respect to their information need and the search process, principles of human communication, and clients' hidden assumptions with respect to information, may facilitate the reference interview and/or help the searcher better understand the complexities of clients' search requests. Additional research is required to more fully identify and validate the fundamental aspects of each discourse that professional searchers appear to navigate. The question is: What fundamental aspects, or types of knowledge, about discourses facilitates search term selection and search effectiveness? We need to identify and validate the aspects that facilitate search term selection across all discourses that professional searchers appear to navigate.

In conclusion, there often are many alternative search terms for a topic because the same topic may be talked of differently in various discourses, and these discourses may change over time. In this study, we show that searchers did not only translate clients' words into search terms appropriate for the IR system, but they also consider how the topic is discussed (or represented) in a variety of discourses, including controlled vocabulary, indexing practice, databases, documents and domains, and their own previous search experience. Searchers appear to evaluate and synthesize the differences among these discourses when selecting search terms. Individual searchers appear to traverse these discourses dynamically, shifting opportunistically among discourses and omitting some discourses. Conceptualizing the selection of search terms as a meeting place of different discourses extends the traditional translation model. It emphasizes the multiplicity and complexity of sources of search terms, the dynamic nature of the search term selection process, and the complex analysis and synthesis of differences and similarities among sources of search terms. We believe that this kind of orientation is needed in quickly changing environments, including the Internet search environment. It may help us develop better education strategies for professional searchers. It may help us design tools that can more effectively support users, perhaps, by proactively sharing information about relevant discourses during the online search process.

Further research is needed to validate and extend the proposed model. To validate the proposed model, the study reported here needs to be replicated, incorporating search requests from different domains and including searchers from additional environments. To extend the model, we need to investigate the online stage of the search process and incorporate the results into the model. Future research should also include investigating which fundamental aspects, or types of knowledge, about discourses facilitate search term selection and search effectiveness.

\section{Acknowledgments}

We thank Claudia Gollop, Bob Losee, Mohan Tatikonda, Barbara Wildemuth, Jeanine Williamson, and the anonymous reviewers for their comments on earlier versions of this article.

\section{Appendix A: The Search Requests Used in the Study}

The search requests used in the study are based on real search requests received at various libraries. Each request has been translated from Finnish.

1) Refugees as an international problem

2) The naval strategies of the U.S. and the Soviet Union 
with respect to the military security of the Northern seas

3 ) Border trade between Finland and the Soviet Union

4) The influence that alternative activities and environmental movements have on social decision-making and the use of power and on solving global environmental problems

5) Independence movements by the Kurds in Turkey

6) Integration of Europe

7) Women as candidates, votes received by women candidates, and women's voting behavior in Finland in parliamentary elections in the $1980 \mathrm{~s}$

8) National groups and ethnic conflicts in the Third
World and their influence on the activities of international organizations

9) Finnish peace-keeping troops in Namibia

10) Social problems, alienation from society, and structural change in society, and their influence on the control people have over their everyday lives in an affluent society

11) Press subsidies in Finland, Sweden, Norway, and Denmark and their influence on the profitability of party newspapers, and the attitudes of the citizens towards financial support given to political parties, particularly towards the press subsidies

12) Islam in international politics

\section{Appendix B: Categories for Sources of Search Terms}

\begin{tabular}{|c|c|c|}
\hline Category & Working defintion & Examples \\
\hline Controlled vocabulary & $\begin{array}{l}\text { When searchers described how they used a controlled } \\
\text { vocabulary to find search terms }\end{array}$ & $\begin{array}{l}\text { "I looked at the controlled vocabulary ...," } \\
\text { "It was in the vocabulary ...," } \\
\text { "I used this vocabulary ...," } \\
\text { "The vocabulary gave it . ..", }\end{array}$ \\
\hline Documents & $\begin{array}{l}\text { When searchers spoke about how they thought about } \\
\text { the words which may occur in documents in the } \\
\text { domain }\end{array}$ & $\begin{array}{l}\text { "The title could be ...", } \\
\text { "The author of a book might have used these words ..., } \\
\text { "In the literature, it might be spoken about ..." }\end{array}$ \\
\hline Indexing practice & $\begin{array}{l}\text { When searchers described how they were thinking } \\
\text { about the way indexers would have used index } \\
\text { terms }\end{array}$ & $\begin{array}{l}\text { "Maybe the book is indexed by these words ...", } \\
\text { "An indexer should have noticed to use this word ...," } \\
\text { "Indexers usually use these words as index words ...," } \\
\text { "If there is this aspect in a book, it is always indexed with this } \\
\text { word ...", }\end{array}$ \\
\hline Clients' search request & $\begin{array}{l}\text { When searchers explained that they used the same } \\
\text { word the client had used }\end{array}$ & $\begin{array}{l}\text { "These words came from the search request." } \\
\text { "These words were used already in a search request.," } \\
\text { "I saw this word in the search request.", }\end{array}$ \\
\hline Database & $\begin{array}{l}\text { When searchers reported they thought about the } \\
\text { content and structure of a database when making } \\
\text { decisions about search terms }\end{array}$ & $\begin{array}{l}\text { "Because the database is so small I need many alternatives } \\
\text { "..", } \\
\text { "Index terms are enough for this topic in this database ...", } \\
\text { "Because of the structure of the database, I tried to imagine } \\
\text {..." } \\
\text { "In the database, there exists/does not exist ...", }\end{array}$ \\
\hline $\begin{array}{l}\text { Searchers' previous } \\
\text { search experience }\end{array}$ & $\begin{array}{l}\text { When searchers reported that their selection of terms } \\
\text { was based on what they had found and learned } \\
\text { earlier during an earlier search process }\end{array}$ & $\begin{array}{l}\text { "I have a long search experience. Therefore, I prefer these } \\
\text { specific search terms ...," } \\
\text { "I have searched this topic earlier and I know ...," } \\
\text { "My previous search experience was that it difficult to find } \\
\text { good search terms for this topic. I tried to remember...," }\end{array}$ \\
\hline
\end{tabular}

\section{References}

Abercrombie, N., Hill, S., \& Turner, B. S. (1994). The Penguin dictionary of sociology (3rd ed.). London: Penguin Books.

Barry, C. (1994). User-defined relevance criteria: An exploratory study. Journal of the American Society for Information Science, 45, 149159.

Bates, M. J. (1990). Where should the person stop and the information search interface start? Information Processing and Management, 26(5), 575-591.

Bates, M. J., Wilde, D. N., \& Siegfried, S. (1993). An analysis of search terminology used by humanities scholars: The Getty online searching project report number 1. Library Quarterly, 63, 1-39.

Berg, B. L. (1989). Qualitative research methods for the social sciences. Boston: Allyn and Bacon.
Bloom, E. (Ed.) (1956). Taxonomy of educational objectives. New York: David Mckay.

Borland, R. J. (1987). "The in-formation of information systems" in R. J. Borland \& R. A. Hirschheim (Eds.), Critical issues in information systems research (pp. 363-380). New York: John Wiley \& Sons.

Fidel, R. (1986). Towards expert systems for selection of search keys. Journal of the American Society for Information Science, 37, 37-44.

Fidel, R. (1991a). Searchers' selection of search keys: I. The selection routine. Journal of the American Society for Information Science, 42, 490-500.

Fidel, R. (1991b). Searchers' selection of search keys: II. Controlled vocabulary or free-text searching. Journal of the American Society for Information Science, 42, 501-514.

Fidel, R. (1991c). Searchers' selection of search keys: III. Searching 
styles. Journal of the American Society for Information Science, 42, 515-527.

Hjerppe, R. (1996). Go with the flow, or abide by the side, or watch the waves? Challenges of change for knowledge organization. In R. Green (Ed.), Knowledge organization and change (pp. 10-25). Frankfurt/Main: Indeks Verlag.

Iivonen, M. (1990). Interindexer consistency and the indexing environment. International Forum on Information and Documentation, 15(2), 16-21.

Iivonen, M. (1994). Challenges and opportunities of libraries as online search environments. Libri, 44(1), 28-46.

Iivonen, M. (1995). Consistency in the selection of search concepts and search terms. Information Processing \& Management, 31(2), 173-190.

International Organization for Standardization. (1985). Documentation-Methods for examining documents, determining their subjects, and selecting indexing terms (ISO 5963). Geneva, Switzerland: Author.

Lancaster, F. W. (1972). Vocabulary control for information retrieval. Washington, DC: Information Resources Press.

Leonard, L. E. (1977). Inter-indexer consistency studies, 1954-1075: A review of the literature and summary of study results. Champaign, IL: University of Illinois.

Markey, K. (1984). Interindexer consistency tests: A literature review and report of a test of consistency in indexing visual materials. Library and Information Science Research, 6, 155-167.

Rasmussen, J., Pejtersen, A. M., \& Goodstein, L. P. (1994). Cognitive systems engineering. New York: Wiley.

Saracevic, T., Mokros, H., Su, L. T., \& Spink, A. (1991). Interaction between users and intermediaries in on-line searching. In M. E. Wil- liams (Ed.), Proceedings of the 12th National On-Line Meeting1991 (pp. 329-341). Medford, NJ: Learned Information.

Schamber, L., \& Bateman, J. (1996). User criteria in relevance evaluation: Toward development of a measurement scale. In S. Hardin (Ed.), Proceedings of the 59th Annual Meeting of the American Society for Information Science (pp. 218-225), Medford, NJ: Information Today, Inc.

Schön, D. A. (1983). The reflective practitioner: How professionals think in action. New York: Basic Books.

Shute, S. J., \& Smith, P. J. (1993). Knowledge-based search tactics. Information Processing and Management, 29(10), 29-45.

Simon, H. A. (1981). The sciences of the artificial. Boston: MIT Press. Sonnenwald, D. H. (1993). Communication in design. Unpublished doctoral dissertation, Rutgers, The State University of New Jersey, New Brunswick, NJ.

Sonnenwald, D. H., \& Pejtersen, A. M. (1994). Towards a framework to support information needs in design: A concurrent engineering example. In H. Albrechtsen \& S. Oernager (Eds.), Advances in knowledge organization: Vol. 4 (pp. 161-172). Frankfurt/Main: Indeks Verlag.

Spink, A., \& Saracevic, T. (1997). Interaction in information retrieval: Selection and effectiveness of search terms. Journal of the American Society for Information Science, 48, 741.

$\mathrm{Su}, \mathrm{L} . \mathrm{T}$. (1991). An investigation to find appropriate measures for evaluating interactive information retrieval. Unpublished doctoral dissertation, Rutgers, The State University of New Jersey, New Brunswick, NJ.

$\mathrm{Su}, \mathrm{L}$. T. (1996). Value of search results as a whole as a measure of information retrieval performance. Proceedings of the 59th ASIS Annual Meeting, 33, 226-237. 